Nonlinear Processes in Geophysics (2005) 12: 345-352

SRef-ID: $1607-7946 / \mathrm{npg} / 2005-12-345$

European Geosciences Union

(c) 2005 Author(s). This work is licensed

under a Creative Commons License.

\title{
Holocene climate variability on millennial scales recorded in Greenland ice cores
}

\author{
A. Witt and A. Y. Schumann \\ Nonlinear Dynamics Group, Physics Department, University of Potsdam, PB 601553, 14469 Potsdam, Germany \\ Received: 11 November 2004 - Revised: 13 February 2005 - Accepted: 14 February 2005 - Published: 21 February 2005 \\ Part of Special Issue "Nonlinear analysis of multivariate geoscientific data - advanced methods, theory and application"
}

\begin{abstract}
Climate variability is triggered by several solar and orbital cycles as well as by the intern ocean dynamics. Consequently, paleoclimate proxy records are expected to vary on very different time scales ranging from subdecadal to millennial duration. We demonstrate, that Foster's (Foster, 1996) wavelet analysis technique is an appropriate tool for investigating temporarily changing spectral properties of records characterized by awkward sampling quality, which is a typical feature of climate proxy records. By applying it to the Holocene part of different glaciochemical records of Greenland ice cores we proof evidence for a significant contribution of the 1.47 kiloyears cycle over alomst the entire Holocene.
\end{abstract}

\section{Introduction}

The ice sheet over Greenland consists of continously deposited and over a time period of more than 250000 years accumulated snow. Climate conditions have controlled the texture of the precipitated snow and that of the surface layer, such that annual layers and patterns within this sequence of layers are arisen. Hence, ice cores drilled in Greenland provide detailed information about paleoclimate and its variability. Many core properties which appeared to be similar for different coring locations account for geographically extended, i.e. North Atlantic, climatic patterns and climatic evidence of their variations (Grootes et al., 1993; Taylor et al., 1993). In the uppermost core part decadal or better resolution of important characteristics can be achieved. The deeper the snow/ice is located the stronger mass pressure and internal ice sheet dynamics have been taken effect, resulting in depletion, pinching and swelling of the layers. Thus, equidistant sampling of core properties along the depth of a core leads to a coarsening of time resolution with increasing age, corresponding sampling intervals might cover time periods

Correspondence to: A. Witt

(awitt@agnld.uni-potsdam.de) up to several hundred years. The analysis presented here investigates cyclicity of the ice core patterns evolved during the Holocene utilizing its high time resolution.

As explained above ice cores contain fossil water, and thus they have conserved the isotopic signature and chemical composition of the precipitation over Greenland at its deposition time. Moreover, these characteristics indicate former climatic conditions and variability. Consequently, variations on very different time scales are expected to be found: The orbital parameters of the Earth change on Milankovitch scales (19, 23, 41 and 100 thousand years); the solar output varies not only according the famous 11 -years cycle, but also with periods of 22, 89, 148 and 207 years or 1800-2500 years (Sonnet et al., 1984) respectively; its modulation by the solar internal motion shows cycles on decadal scales (Palus et al., 2000); ocean and ice dynamics (Stuiver and Braziunas, 1993 ) is related to variability of 512 years, 1.47 kiloyears and 6 kiloyears; changes in atmospheric loading (O'Brien et al., 1995) are tied to a period of almost 3 kiloyears and climate oscillations such as the El Niño Southern Oscillation or the North Atlantic Oscillation (Sonnet et al., 1984) show variability on a (sub-)decadal time scale.

These records of isotopic and chemical composition together with nonlinear age-depth models, transforming equidistant core-depth sampling into non-equidistant age sampling, represent reference data sets that provide information about late Quaternary climate conditions and climate variability in the North Atlantic region (Mayewski et al., 1997, 1990; Taylor et al., 1996; Yang et al., 1995; Grootes et al., 1993). Among several records the most prominent one, the $\delta^{18} \mathrm{O}$ record, serves as a paleo temperature proxy.

In first order approximation low $\delta^{18} \mathrm{O}$ values are related to cold periods (glacials) and high amplitudes indicate higher air temperatures over the North Atlantic. The main revelation of this record has been the detection of DansgaardOeschger (Dansgaard/Oeschger et al., 1984) events, i.e. very rapid warmings followed by a gradual cooling and a final return to glacial temperatures during the last glacial (100000 until 11800 years ago) in Greenland. 
Strong fluctuations of the $\delta^{18} \mathrm{O}$ concentration have been accompanied by significant changes in accumulation rate and dust content. Mentioned temperature changes reflect the switch on/switch off dynamics of the North Atlantic thermohaline ocean circulation. During a switch-on-period highly dense, i.e. cold and saline, water in the Labrador and Norwegian sea sinks to depths. The loss of water is compensated by poleward movement of warm, saline surface water from low latitudes to the North Atlantic or the Gulf stream, respectively. When the Gulf stream water has been cooled down and become dense, the circuit is closed. This circulation transports a remarkable quantity of heat to the surface of the North Atlantic, that increases primarily the air temperature and changes secondly the atmospheric circulation patterns. When the water density gradients in the Labrador and Norwegian fall below a critical threshold, this circulation is ceased. It starts again as soon as this gradient increases to a level where density-induced overturning of the water column can resume (for an overview and a list of references see Bradley, 1999).

Detailed spectral analysis of the Greenland ice sheet $\delta^{18} \mathrm{O}$ record has proven a 1470 year cycle, that is interpreted as the preferred recurrence time of Dansgaard-Oeschger events. Although the Holocene part of the record does not show that cycle, ample evidence of its existence over the past 15000 years has been found in sediments of deep sea cores from the North Atlantic (Bond et al., 1997, 2001). The grain size distributions found in the sediment and diatom tests prove advection of cool, ice-bearing water from Island to the south with a cyclicity close to 1470 years.

The intention of this paper is to examine accumulation rate, $\delta^{18} \mathrm{O}$ and chemical composites of Holocene sections of Greenland Ice cores in order to search for indications of oscillations on the Dansgaard-Oeschger scale. Since the amplitude of this millennial scale period cannot assumed to be constant, wavelet analysis, which allows a simultaneous decomposition in time and frequency domain, is applied.

The paper is organized as follows: First methods and results regarding data analysis of ice core $\delta^{18} \mathrm{O}$ records are resumed. A discussion about awkward sampling properties of measured ice core data is followed by a presentation of Foster's wavelet analysis algorithm as a reliable technique to analyze such data. The results concerning millennial scale variability of the Greenland Ice cores are presented and discussed and finally an outlook on methodical enhancements of the presented technique is given.

\section{Data analysis of the $\delta^{18} \mathrm{O}$ records from Greenland}

Period analysis of unevenly sampled data has been introduced in the context of astrophysical observations (Lomb, 1976; Scargle, 1982; Foster, 1995). Applying these algorithms to perform a spectral analysis a sharp peak centered around a period of 1470 years has been found in different ice (Grootes et al., 1993; Mayewski et al., 1997; Schulz, 2002a) and ocean (Bond et al., 1997) cores. Since no orbital pace- maker acting on this period is known, the significance as well as the origin of the peak have been discussed intensively: The hypothesis of Wunsch (Wunsch, 2000) that this peak is an alias of the annual cycle due to sampling on larger time scales initiated a controversial discussion (Meeker et al. and Wunsch, 2001). By now it is generally accepted that this peak is a signature of the ocean ice dynamics. Schulz (Schulz, 2002b) has proven that the significant strength of the peak at the 1470 year period (with respect to the $\delta^{18} \mathrm{O}$ record of Greenland Ice Sheet Project 2 (GISP2) campaign) is caused by the age interval 31-36 kiloyears B.P. (B.P. means before present and present is dated with 1950). Further, he could show that most time intervals between adjacent Dansgaard-Oeschger events are integer multiples of 1470 years which is a clear indicator of a pacemaker. Rahmstorf has demonstrated that the timing of these climate events follows a precise clock (Rahmstorf, 2003). However, the origin of this pacemaker is still unclear. Recent numerical simulation of paleo climatic conditions led to the assumption the peak is caused by superposition of two solar cycles and a suitable offset (Braun et al., 2004). Fourier analysis needs stationary records as a prerequisite, i.e. considered records are supposed to have a time independent mean value and autocorrelation function, or, in other words, the amplitudes of different periods remain constant during the complete observational epoch. The ice core records, however, do not fulfill this conditions. Since wavelet analysis delivers a decomposition in time and frequency domain simultaneously it seems to be the more appropriate tool to characterize time dependent variability.

\section{Climate proxy data are awkward data}

From the viewpoint of statistical data analysis climate proxy data based on lab measurements of ice cores are data with awkward sampling properties:

- Isotope-based age estimates that are affected by random and systematic errors cause uncertainties concerning the age axis. This property makes paleo climatic records fundamentally different to all data resulting from direct instrumental measurements as instrumental climate data, output of physical lab experiments or even medical data as ECG/EEG measurements. Moreover, these uncertainties along the time axis, i.e. the standard deviation of the random error concerning the estimated ages, grow backwards in time. An appropriate data analysis has to be performed.

- Due to the nonlinear age-depth relation the data are unevenly sampled. Further, the mass pressure of the ice leads to an increasing compression with growing overburden or age, hence, to a strong depletion of the lower, i.e. older, part of the ice sheet. Laboratory measurements are performed for core slices of constant thickness; consequently the data density (data per age interval) in the older part diminishes dramatically. 
- As usual, amplitudes are disturbed by noise. The noise intensity of the GISP2 data varies for different paleo climatic periods and is further affected by changing sampling rates.

Standard time series analysis techniques and related software packages require evenly sampled and stationary time series, and implicitly, a well-defined time axis. Since data under consideration do not fulfill these assumptions alternative techniques are required. The problem concerning uneven sampling and non-stationarity can be solved by implementing Foster's wavelet analysis algorithm. He considers the wavelet analysis for a fixed localization centered around the age $\tau$ and concerning to the period $\omega$ as a weighted projection of the time series onto a function space spanned by the sine/cosine basis function related to $\omega$ and the constant function.

\section{Fourier and wavelet analysis of unevenly spaced time series}

In this section the main mathematical ideas behind the Fourier and wavelet transform for unevenly sampled data are outlined.

Besides its well known physical interpretation the Fourier transform can be considered from the viewpoint of functional analysis as a main axis transformation. To accomplish this more detailed some notations have to be introduced. A possibly unevenly sampled time series $\boldsymbol{x}=\left\{x\left(t_{j}\right)\right\}_{j=1}^{N}$ is considered as a point of the $N$-dimensional sampling space that is spanned by the standard basis $\delta_{\mathbf{k}}=\left\{\delta_{t_{j}, t_{k}}\right\}_{j=1}^{N}, k=1, \ldots, N$, where $t_{j}$ denotes the (estimated) age of the $j$-th measured point, $x\left(t_{j}\right)$ stands for its amplitude, and $\delta_{t_{j}, t_{k}}$ is the Kronecker delta. For evenly spaced time series a scalar product is introduced by

$$
\langle\boldsymbol{x}, \boldsymbol{y}\rangle=1 / N \sum_{j=1}^{N} x\left(t_{j}\right)^{*} y\left(t_{j}\right),
$$

which implies the definition of a norm $\|\boldsymbol{x}\|^{2}=\left\langle\boldsymbol{x}^{*}, \boldsymbol{x}\right\rangle$, where $\boldsymbol{x}^{*}$ is the conjugate complex of $\boldsymbol{x}$. A discrete Fourier transformation can be understood as a set of projections onto subspaces that are spanned by the constant vector $\boldsymbol{\Psi}_{1}=1=\left\{1\left(t_{j}\right)\right\}_{j=1}^{N}, 1\left(t_{j}\right)=1 \forall t_{j}$ and a complex Fourier basis vector $f\left(\omega_{k}\right)=\left\{e^{i \omega_{k} t_{j}}\right\}_{j=1}^{N}$ which one can decompose in its real and imaginary parts, i.e. $\Psi_{2}=\left\{\cos \left(\omega_{k} t_{j}\right)\right\}_{j=1}^{N}$ or $\boldsymbol{\Psi}_{3}=\left\{\sin \left(\omega_{k} t_{j}\right)\right\}_{j=1}^{N}$, respectively. In case of even sampling intervals $\Delta t$, i.e. $t_{j}=j \Delta t$, the basis functions $\boldsymbol{\Psi}_{2,3}\left(\omega_{k}\right)$ are orthogonal if $\omega_{k}=\frac{2 \pi k}{N \Delta t}$ for $1 \leq k \leq \frac{1}{2} N$ manifesting the interpretation as a main axis transformation. This orthogonality gets lost when considering unevenly spaced data sets.

Foster (Foster, 1995) has proposed to determine the projection coefficients $\tilde{y}^{a}, a=1,2,3$ which correspond to the chosen period $\omega_{k}$ in such a way that the optimum projection is realized, i.e. the distance between the original function $\boldsymbol{x}$ and its projection $\left\|\boldsymbol{x}-\left(\tilde{y}^{1} \boldsymbol{\Psi}_{1}+\tilde{y}^{2} \boldsymbol{\Psi}_{2}+\tilde{y}^{3} \boldsymbol{\Psi}_{3}\right)\right\|$ is minimized.
A very similar scheme can be applied to perform a wavelet transformation Foster (1996) (for some more details, please see the Appendix). Therefore, the scalar product as introduced in Eq. (1) needs to be generalized by a weighted scalar product:

$$
\langle\boldsymbol{x}, \boldsymbol{y}\rangle=\frac{\sum_{j=1}^{N} w_{j} x\left(t_{j}\right) y\left(t_{j}\right)}{\sum_{j=1}^{N} w_{j}} .
$$

The Fourier transformation is equivalent to $w_{j}=1, j=1, \ldots, N$. For a wavelet transformation, the weights $w_{j}$ should focus the consideration onto data points located in a neighborhood of the age $\tau$. For the abbreviated Morlet-Wavelet $f(t)=e^{-c \omega^{2}(t-\tau)^{2}} \cdot e^{i \omega(t-\tau)}$ used here the weights are defined as $w_{j}=\exp \left\{-c \omega^{2}\left(t_{j}-\tau\right)^{2}\right\}$ for discrete times $t_{j}$, where $c=\left(8 \pi^{2}\right)^{-1}$.

The projection onto the subspaces, which are spanned by the basis $\left\{\left\{1\left(t_{j}\right)\right\}_{j=1}^{N},\left\{\cos \left(\omega\left(t_{j}-\tau\right)\right)\right\}_{j=1}^{N}\right.$, $\left.\left\{\sin \left(\omega\left(t_{j}-\tau\right)\right)\right\}_{j=1}^{N}\right\}$, is defined in the same way as above and the determination of its coefficients is equivalent, too. Please note that the effective number of data points that contribute to the projection shrinks to

$N_{\text {eff }}=\frac{\left(\sum_{j=1}^{N} w_{j}\right)^{2}}{\sum_{j=1}^{N} w_{j}^{2}}$,

that is in any case less or equal the number of time series elements $N$.

Finally, a statistical test is implemented adopting a Null hypothesis. This Null hypothesis is related to a model function which we consider being pure white or colored noise. Foster has introduced a $Z$-statistic based on a $F$-Test, which evaluates the normalized quotient of the (weighted) variation of the projected data and the projected model function of pure noise. The degrees of freedom mainly depend on the effective number of data points $N_{\text {eff. }}$. This can lead to different degrees of freedom for identical frequencies $\omega$ but different sampling rates. For each pair $\omega, \tau$ the hypothesis is tested, and, if rejected, the period- $\omega$ contribution to the variability near the age $\tau$ is interpreted as a climatic signal beyond the background noise. The exact formalism is outlined in the Appendix or can be read in more detail in Foster (1996).

\section{Results and discussion}

We have focused our considerations on centennial and millennial scale variability of different physical and chemical properties of the GISP2 data set, that can be downloaded from www.ngdc.noaa.gov/paleo/icecore/greenland/ summit/document/gispdata.htm. The analysis has been concentrated on the Holocene and its termination, i.e. the time interval of the past 15700 years.

Since spectral and more sophisticated analysis of the $\delta^{18} \mathrm{O}$ record has provided variability on the 1.47 kiloyears scale over the last glacial it has been the object of our first attempt to search for such variability in the younger part. In 

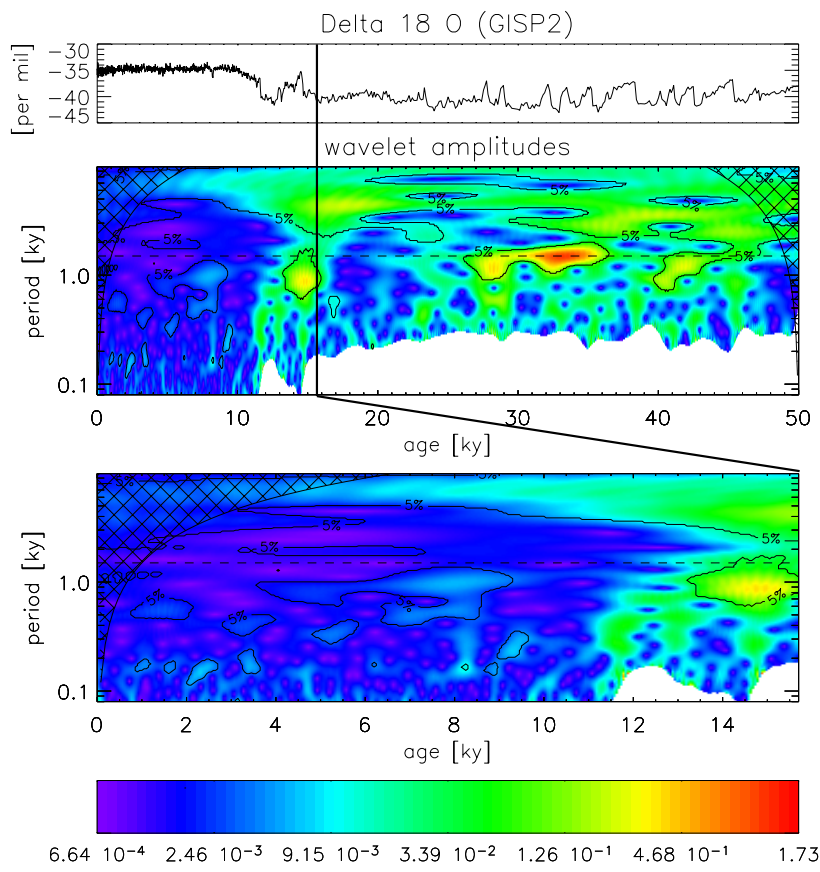

Fig. 1. Wavelet analysis of the GISP $2 \delta^{18} \mathrm{O}$ record (top): The second and third panels visualize the wavelet amplitudes depending on the localization (age) and the period length for the past 50000 years as well as the Holocene part of the record. White areas are related to effective number of data points less than 6 , where amplitudes cannot be estimated reliably. Cross hatched regions indicate the cone of influence, where the wavelet analysis is affected by edge effects. Contour lines mark periodic shares that are significantly different from a red noise background assuming an error of the first type of $5 \%$. The bottom panel display the color codes. The dashed line stands for the 1.47 kiloyears cycle.

order to prove the performance of the wavelet technique the 50 kiloyears history of the $\delta^{18} \mathrm{O}$ has been analyzed. As can be seen in Fig. 1 (second panel) the wavelet analysis reproduces results of former investigations (see Sect. 1) and brings out evidence for significant variations on the 1.47 kiloyears scale for ages from 26 to 37 kiloyears and from 40 to 48 kiloyears. Slightly increased amplitudes on that scale are visible throughout almost the entire glacial. The (relatively) low data density may be the reason why the statistical test cannot significantly distinguish this increased amplitudes from the red noise background. Surprisingly, the results show, that the Holocene section contains mainly red noise (third panel) and centennial as well as millennial scale variability for some shorter time intervals. Neither a continuous periodic portion on the 1.47 kiloyears scale nor even a shorter period does exist. What are the reasons? Changes in $\delta^{18} \mathrm{O}$ ice cores are rather rough indicators of North Atlantic sea surface temperature variation, because precipitation over Greenland has different sources namely the North Atlantic and the North Pacific (Kiefer et al., 2002). When the sea surface temperature in the North Pacific region is higher than in the North Atlantic the variations in $\delta^{18} \mathrm{O}$ are faded or disappear at all. However, the analysis reveals a contribution with a period length

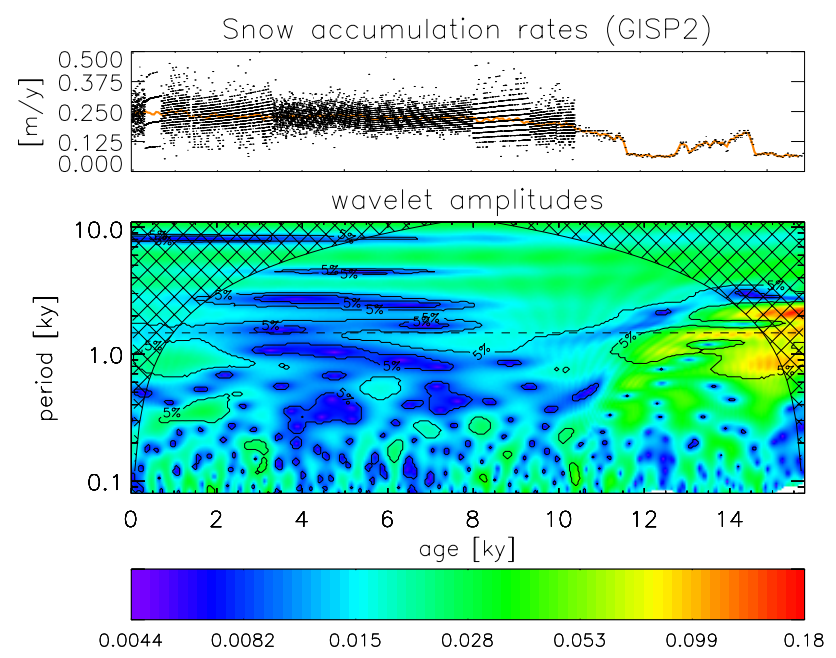

Fig. 2. The GISP2 accumulation rate record (top) and its 100-year mean (red). The lower panel shows the wavelet amplitudes, contoured regions contain periodic shares significantly different from red noise assuming $5 \%$ error probability.

of 980 years (3.5 kiloyears until 9 kiloyears) which was recently discussed by (Schulz, 2002a) as signature of the arctic signal and a second one (3 kiloyears until 7 kiloyears) on a 2.4 kiloyears scale, which is a scale on that the solar output changes (Stuiver and Braziunas, 1993).

However, Greenland ice core records are composed of many characteristic parameters, some among them are interpreted as proxies for sea surface temperature, too. Snow accumulation rates (Cuffey et al., 1995; Cuffey and Clow, 1997) which are primarily a precipitation proxy reflect the humidity of the ancient atmosphere. Since the formation of moisture rises by increasing temperatures, changes in snow accumulation rates can be understood as temperature driven, i.e. this data set turns out to be an alternative to the $\delta^{18} \mathrm{O}$ record. The GISP2 accumulation rates have been modeled by a combined heat- and ice flow model constrained by measurements of the borehole temperature, the $\delta^{18} \mathrm{O}$ record and the age depth model of that ice core. Figure 2 that presents this record and its wavelet transform gives clear evidence that the humidity of the atmosphere has been changed on millennial scales, and in particular on the 1.47 kiloyears scale. Variability on this scale can be found for ages between 5 and 10 kiloyears B.P., during the early Holocene (more than 12 kiloyears ago) and with rather high amplitudes during the Holocene's termination. For the time period 28.5 kiloyears B.P. the wavelet amplitudes show well defined bands on 1.8-2.3 and 3-4 kiloyears. Throughout the past 11 kiloyears amplitudes are amplified on broad band between 5 and 7 kiloyears which seems to be tied to the mechanism responsible for Heinrich events (McManus et al., 1999).

The several dissolved chemical constituents of ice cores serve as finger prints of both, the source area as well as the pathway of the air masses that ejected precipitation over Greenland. Ammonium is a tracer of continental (North 


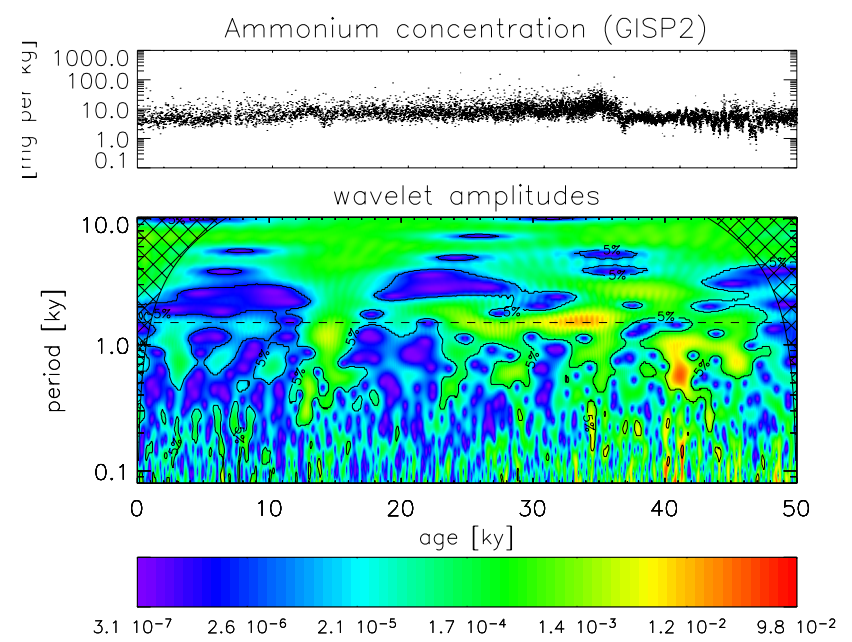

Fig. 3. The GISP2 ammonium concentration record (top) and its wavelet amplitudes (bottom). Contoured regions contain periodic shares significantly different from red noise assuming an error probability of $5 \%$.

American) bioactivity, and serves as an indicator of biomass burning events (Taylor et al., 1996). High ammonium concentrations in the ice core indicate stronger atmospheric loading over the North American continent, meaning improved transport conditions in terms of increased storminess in this region and/or rather dry climatic conditions. High-frequent storm events are caused by a strengthening of the polar vortex that evokes growing pressure gradients between the pole and lower latitudes and a cooling in the North and at middle latitudes, respectively. Besides of some spread centennial variability the wavelet analysis reveals an almost continuous cycle on the 1.5 kiloyears scale, namely for ages between 2 and 10.5 kiloyears B.P., and, with very high amplitudes from 12.5 to 15.7 kiloyears B.P. (Fig. 3). Further, continuous cycles with periods of 3.0-3.5 kiloyears, 4.5 kiloyears and around 7 kiloyears are visible, the first two of them are close to multiples of the famous period of 1.47 kiloyears. Rather similar results are found for the variations in Potassium (Witt and Oberhaensli, 2005) ${ }^{1}$, which is related to the vegetation cover of the soil.

Besides of its contribution to the analysis of non-stationary frequency structures, wavelet analysis can be used to evaluate wavelet phases (see Eq. A11) and to band-pass-filter the considered record. The filtered series is nothing as the product $A(\omega, \tau) \sin (\Phi(\omega, \tau))$ for a fixed frequency $\omega$. Since significance for variability on 1.47 kiloyears scales has been proven for both, the ammonium as well as the precipitation rate record, which both serve as proxies for warmer/cooler climatic conditions, the phase relation between the 1.47 kiloyears-portions of these records have been investigated. The computations show (see Fig. 4) a con-

\footnotetext{
${ }^{1}$ Witt, A. and Oberhaensli, H.: Millennial Scale Variability of Atmospheric Loading throughout the Holocene, in preparation (2005).
}
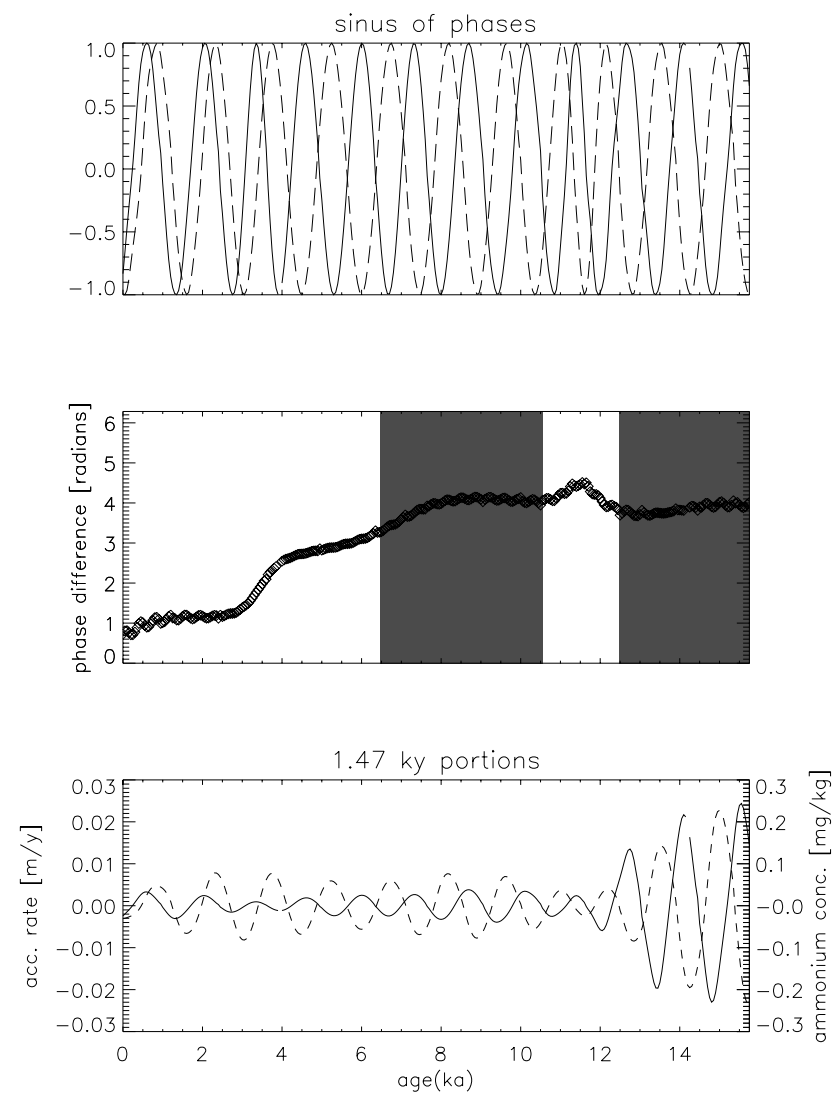

Fig. 4. Consideration of the 1.47 kiloyears portions of the ammonium and the accumulation rate records: phases (top), phase differences and 1.47 kiloyears portions of both records. Gray background indicates significant periodicity on the 1.47 kiloyears scale for both records.

stant phase shift during time periods where the 1.47 kiloyears amplitudes of both data sets are significantly different from the noisy background, i.e. between 6 and 10.5 kiloyears B.P. and older than 12 kiloyears B.P. These phase shifts concentrate near $4 /(2 \pi)$ which is $60 \%$ of a period length, meaning the ammonium variation is approximately 1 kiloyear lagged behind the accumulation rate. This phase shift can be explained in terms of different temperature codings of the two records: the accumulation rate goes in paralleled with temperature variations while high ammonium concentration stands for increased storminess which, as explained above, is accompanied by low temperatures at middle and higher latitudes. Considering the moisture content of the atmosphere, high precipitation values are related to a humid atmosphere, whereas massive biomass burning (high ammonium concentration) requires dryer conditions.

\section{Conclusion and outlook}

The ice core data are undoubtedly records of highly nonstationary and nonlinear processes. Their wavelet analysis pro- 

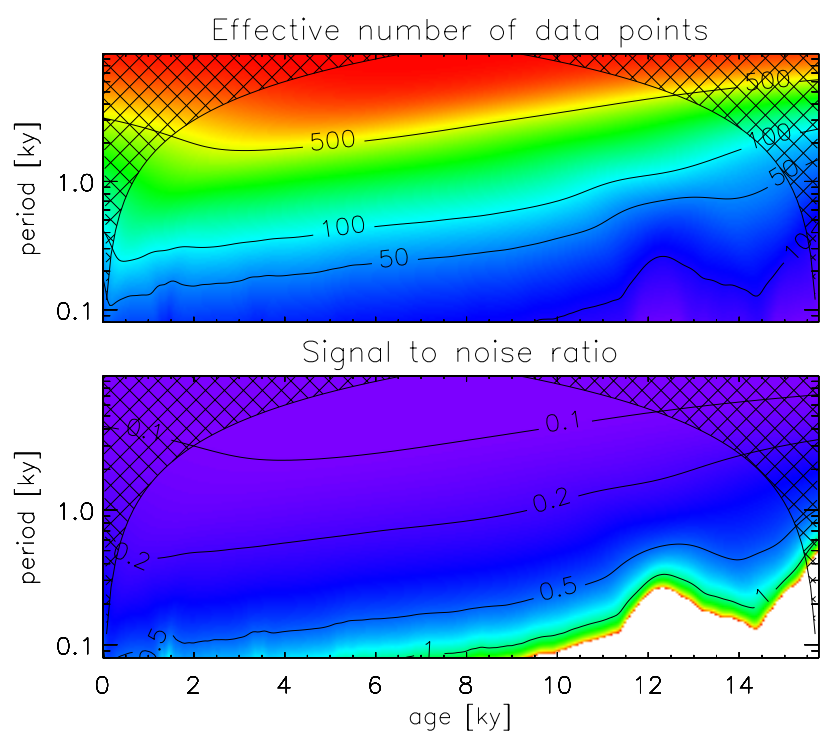

Fig. 5. The top panel presents the effective number of data points concerning the wavelet plot of Fig. 1, in dependence on the localization (age) and the period length. The bottom panel shows the signal to noise ratios that have to be passed over to detect periodic dynamics on the related scale and localization. White color stands for effective number of data points less than 10 .

vides information about the different periodic components and their time dependent amplitudes.

The uneven time sampling of these records, which was the strongest limit of the applicability of standard time series analysis techniques, has been overcome by adopting Foster's wavelet algorithm. But how to deal with the uncertainties along the age axis? All wavelet plots presented show extended areas of variability that is significantly different from the noisy background. A moderate alteration of the age axis would result in very similar wavelet amplitudes, most significant structures are resistant to slightly modified ages. As long as the periods under consideration remain significantly larger than the expected uncertainties of the ages, the proposed method is an adequate tool to understand the structure of the data.

A special peculiarity of the GISP2 record is the coarsening of the sampling rate with the age that is equivalent to a noise growth. In other words the data quality is inhomogeneous and tendentious worsen for the older part. Regarding the wavelet analysis as a statistical hypothesis test against red or white noise, rough sampling is equivalent to large errors of the second type, i.e. cyclicity that is present but cannot be detected. This error of second type is closely related to the effective number of data points which depends on the localization and period length of the considered wavelet. In Fig. 5 the effective number of data points concerning the $\delta^{18} \mathrm{O}$-record (Fig. 1) is presented. Further, the signal to noise ratios, i.e. the quotient of the standard deviations (concerning white noise), that have to be passed over in order to descry the periodic structures hidden in the noise by means of the method used in this paper, are shown. Both plots show struc- tures that depend not only on the period length as it would be in the case of evenly spaced data, but also on the localization. This emphasizes the need for a careful interpretation of the results of a wavelet analysis, in particular for short-scale variability. On the other hand it points out, that millennial scale variability is rather robust against the sampling properties, because it is detectable through the whole record, wherever its signal to noise ratio is larger than 0.2.

The hypothesis against which we are testing by applying the technique described above is "The record is red noise". This red noise, however, is modeled in a rather simple way as the realization of a first order autoregressive process. Future work has to consider more complicated red noise models, at least higher order autoregressive processes. We have to develop algorithms that fit coefficients belonging to such noise models.

Since paleo climatic records are in general not Gaussian distributed and climate variations are neither symmetric in time nor in amplitude, a sinusoidal wavelet basis as we have chosen it does not seem to be ideal. In the framework of a forthcoming paper we are going to generalize our approach to more complicated model functions.

\section{Appendix - Wavelet analysis of unevenly sampled data in formulae}

Wavelet analysis is basing on wavelet functions, so called mother wavelets. In this contribution we have applied the abbreviated Morlet-Wavelet

$f(t)=e^{-c \omega^{2}(t-\tau)^{2}+i \omega(t-\tau)}=e^{-c \omega^{2}(t-\tau)^{2}} \cdot e^{i \omega(t-\tau)}$,

a function that depends on the period $\omega$ and the considered time $\tau$ and which is rapidly decreasing with growing distance to $\tau$. The parameter $c$ is chosen equal to $\left(8 \pi^{2}\right)^{-1}$. To consider the wavelet transform as a weighted projection from the sampling space into a lower dimensional function space, a statistical weight and a basis of this function space need to be defined. The statistical weights are chosen in accordance to the first factor of Eq. (A1), i.e.

$w_{j}=e^{-c \omega^{2}\left(t_{j}-\tau\right)^{2}}$

and contribute to a weighted scalar product

$\langle\boldsymbol{x} \mid \boldsymbol{y}\rangle=\frac{\sum_{j=1}^{N} w_{j} x\left(t_{j}\right) y\left(t_{j}\right)}{\sum_{j=1}^{N} w_{j}}$.

This enables to write the (weighted) variation of the sampling space vector $\boldsymbol{x}$, i.e. of the time series as:

$V_{x}=\langle\boldsymbol{x} \mid \boldsymbol{x}\rangle-\langle 1 \mid \boldsymbol{x}\rangle^{2}$.

The complex second factor of Eq. (A1) is separated into its real and imaginary part, which gives, together with the constant vector a basis for the projection space (for fixed frequency $\omega$ and time localization $\tau$ ):

$\boldsymbol{\Psi}_{\mathbf{1}}=\left\{1\left(t_{j}\right)\right\}_{j=1}^{N}$ 
$\boldsymbol{\Psi}_{2}=\left\{\cos \left(\omega\left(t_{j}-\tau\right)\right)\right\}_{j=1}^{N}$

$\boldsymbol{\Psi}_{\mathbf{3}}=\left\{\sin \left(\omega\left(t_{j}-\tau\right)\right)\right\}_{j=1}^{N}$.

The matrix of the scalar products of these basis vectors, introduced by Foster as super $\mathbf{S}$ matrix, reads:

$\mathbf{S}=\left\langle\boldsymbol{\Psi}_{a} \mid \boldsymbol{\Psi}_{b}\right\rangle, a=1,2,3$.

Thus, the scalar product of elements of the subspace spanned by these basis vectors simplifies to: $\langle\tilde{\mathbf{y}} \mid \tilde{\mathbf{z}}\rangle=\sum_{a=1}^{3} \sum_{b=1}^{3} S_{a b} \tilde{y}^{a} \tilde{z}^{b}$. From the projection coefficients

$\tilde{y}^{a}=\sum_{b=1}^{3} S_{a b}^{-1}\left\langle\Psi_{a} \mid \boldsymbol{x}\right\rangle$,

where $S_{a b}^{-1}$ is the (a, b)-th element of the inverse super S matrix, we obtain the model function $\mathbf{y}$ and its variation:

$$
\begin{gathered}
\boldsymbol{y}=\left\{y\left(t_{j}\right)\right\}_{j=1}^{N}=\left\{\sum_{a=1}^{3} \tilde{y}^{a} \Psi_{a}\left(t_{j}\right)\right\}_{j=1}^{N} \\
V_{y}=\langle\boldsymbol{y} \mid \boldsymbol{y}\rangle-\langle 1 \mid \boldsymbol{y}\rangle^{2}=\sum_{a=1}^{3} \sum_{b=1}^{3} \tilde{y}^{a} \tilde{y}^{b} S_{a b}-\left(\sum_{b=1}^{3} \tilde{y}^{b} S_{1 b}\right)^{2} .
\end{gathered}
$$

Note that $\left(\tilde{y}^{a}\right)^{2}$ is the squared coefficient belonging to the a-th basis vector. The Null hypothesis "The data record is white noise" can be tested by evaluating the test statistic

$Z(\omega, \tau)=\frac{V_{y}\left(N_{\mathrm{eff}}-3\right)}{2\left(V_{x}-V_{y}\right)}$,

which follows an F-distribution with $\left(N_{\mathrm{eff}}-3,2\right)$ degrees of freedom. While the F-distribution is only defined for integer degrees of freedom, $N_{\text {eff }}$ is usually not an integer. Thus, an evaluation of concerned F-quantiles requires interpolation and implies errors which are large for small $N_{\text {eff }}$ and can be neglected for large $N_{\text {eff. }}$.

A related formula for a test against red noise cannot be derived straight forward, because correlations between neighboring data points have to be taken into consideration. The expectation value of $V_{y}$, that is needed for construction of a test statistic, gets an additional term depending on the covariance function of red noise and the period length. For more details we refer to the master thesis of A.Y.S. (Schumann, 2004).

The projection coefficients concerning the basis vectors $\Psi_{2}$ and $\Psi_{3}$ supply estimates of the wavelet amplitude

$A(\omega, \tau)=\sqrt{\left(\tilde{y}^{2}\right)^{2}+\left(\tilde{y}^{3}\right)^{2}}$

and the wavelet phase

$\phi(\omega, \tau)=\arctan \left(\tilde{y}^{2} / \tilde{y}^{3}\right)$.

Acknowledgements. The authors thank their colleagues from the Nonlinear Dynamics Group and the Geoforschungszentrum Department 3.3 for discussion. The both of us are indebted to $\mathrm{H}$. Vos for a very careful reading of the manuscript and several valuable hints. A. Y. S. thanks Andreas Braunss for discussion. A. Y. S. was supported by the VW Foundation (Grant number I/78218), A. W. has been funded by the Ministry of Science, Research and Culture of the Federative State Brandenburg.

Edited by: M. Thiel

Reviewed by: $\mathrm{H}$. Vos and another referee

\section{References}

Bond, G., Showers, W., Cheseby, M., Lotti, R., Almasi, P., deMenocal, P., Priore, P., Cullen, H., Hajdas, I., and Bonani, G.: A pervasive millenial-scale cycle in North Atlantic Holocene and Glacial Climates, Science, 278, 1257-1266, 1997.

Bond, G., Kromer, B., Beer, J., Muscheler, R., Evans, M., Showers, W., Hoffmann, S., Lotti-Bond, R., Hajdas, I., and Bonani, G.: Persistent Solar Influence on North Atlantic Climate During the Holocene, Science, 294, 2130-2136, 2001.

Bradley, R. S.: Paleoclimatlogy: Reconstructing Climates of the Quarternary. Academic Press, San Diego, 1999.

Braun, H., Christl, M., Ganopolski, A., Rahmstorf, S., Mangini, A., Kromer, B., Roth, K., Kubatzki, C., and Claussen, M.: Solar forcing of Dansgaard-Oeschger events in the coupled climate system model CLIMBER-2, Geophys. Res. Abstr., 6, 04678, 2004.

Cuffey, K. M., Clow, G. D., Alley, R. B., Stuiver, M., Waddington, E. D., and Saltus, R. W.: Large Arctic temperature change at the Wisconsin-Holocene glacial transition, Science, 270, 455-458, 1995.

Cuffey, K. M. and Clow, G. D.: Temperature, accumulation, and ice sheet elevation in central Greenland through the last deglacial transition, J. Geophys. Res., 102, 26383-26 396, 1997.

Dansgaard, W., Johnson, J., Clausen, H. B., Dahl-Jensen, D., Hammer, N., and Oeschger, U.: North Atlantic climatic oscillations revealed by deep Greenland ice cores, Climate Processes and Climate Sensitivity, AGU, Geophys. Monogr., 29, 288-298, 984.

Foster, G.: The cleanest Fourier spectrum, Astrophys. J., 109, 1889-1902, 1995.

Foster, G.: Wavelets for period analysis of unevenly sampled time series, Astronom. J., 112, 1709-1729, 1996.

Grootes, P. M., Stuiver, M., White, J. C. W., Johnson, S., and Jouzel, J.: Comparison of oxigen isotopes records from the GISP2 and GRIP Greenland ice cores, Nature, 336, 552-554, 1993.

Kiefer, T., Lorenz, S., Schulz, M., Lohmann, G., Sarntheim, M., and Elderfield, H.: Response of Precipitaion over Greenland and the adjacent ocean to North Pacific warm spells during DansgaardOeschger stadials, Terra Nova, 14, 295-300, 2002.

Lomb, N. R.: Least-square frequency analysis of unequally spaced data, Astrophys. Spac. Sci., 29, 447-462, 1976.

Mayewski, P. A., Lyons, W. B., Spencer, M. J., Twickler, M. S., Buck, C. F., and Whitlow., S. I.: An ice core record of atmospheric response to anthropogenic sulphate and nitrate, Nature, 346, 554-556, 1990.

Mayewski, P. A., Meeker, L. D., Twickler, M. S., Withlow, S. I., Yang, Q., Lyons, W. B., and Prentice, P.: Major features and forcing of high-latitude northern hemisphere atmospheric circulation using a 110000 -year-long glaciochemical series, J. Geophys. Res., 102, 26345-26366, 1997.

McManus, J. F., Oppo, D. W., and Cullen, J. L.: A 0.5 million year record of millennial scale climate variability of the North Atlantik, Science, 283, 971-974, 1999. 
Meeker, L. D., Mayewski, P. A., Grootes, P. M., Alley, R. B., and Bond, G. C.: Comment on "On sharp spectral lines in the climate record and the millenial peak" and Wunsch, C.: Reply, Paleoceanography, 16, 544-548, 2001.

O'Brien, S. M., Mayewski, P. A., Meeker, L. D., Meese, D. A., Twickler, M. S., and Whitlow., S. I.: Complexity of Holocene climate as reconstructed from a Greenland ice core, Science, 270, 1962-1964, 1995.

Palus, M., Kurths, J., Schwarz, U., Novotna, D., and Charvatova, I.: The solar cycle is weakly synchronized with the solar internal motion, Int. J. Bifurc. \& Chaos, 11, 2519-2526, 2000.

Rahmstorf, S.: Timing of abrupt climate change: a precise clock, Geophys. Res. Lett., 30, 1510, 2003.

Scargle, J. D.: Studies in astronomical time series analysis, II Statistical aspects of spectral analysis of unevenly spaced data, Astrophys. J., 263, 835-853, 1982.

Schulz, M. and Mudelsee, M.: REDFIT: estimating red-noise spectra directly from unevenly spaced paleclimate time series, Computers \& Geosciences, 28, 421-426, 2002.

Schulz, M.: On the 1470 year pacing of Dansgaard-Oeschger warm events, Paleoceanography, 17, doi:10.1029/2000PA000571, 2002 .
Schumann, A. Y.: Master Thesis: Waveletanalyse von Sedimentdaten unter Einbeziehung von Alters-Tiefen-Modellen, University of Potsdam, Germany, 2004.

Sonett, C. P. and Suess, H. E.: Correlation of bristlecone pine ring width with atmospheric ${ }^{14} \mathrm{C}$ variations, Nature, 307, 141-142, 1994.

Stuiver, M. and Braziunas, T. F.: Sun, ocean, climate and atmospheric ${ }^{14} \mathrm{CO}$ an evaluation of causal and spectral relationships, The Holocene, 3, 289-305, 1993.

Taylor, K. C., Hammer, C. U., Alley, R. B., Clausen, H. B., DahlJensen, D., Gow, A. J., and Gunderstrup, N. S., Kipfstuhl, J., Moore, J. C., and Waddington, E. D.: Electrical conductivity measurements from GISP2 and GRIP Greenland ice cores, Nature, 366, 549-552, 1993.

Taylor, K. C., Mayewski, P. A., Twickler, M. S., and Whitlow, S. I: Biomass burning recorded in the GISP2 ice core: A record from eastern Canada?, The Holocene, 6, 1, 1-6, 1996.

Wunsch, C.: On sharp spectral lines in the climate record and the millenial peak, Paloceanography, 15, 417-424, 2000.

Yang, Q., Mayewski, P. A., Whitlow, S. I., Twickler, M. S., Morrison, M. C., Talbot, R. W., Dibb, J. E., and Linder, E.: Global perspective of nitrate flux in ice cores, J. Geophys. Res., 100, 5113-5121, 1995. 